\title{
Modeling and compensation of geometric errors in simultaneous cutting using a multi-spindle machine tool
}

\begin{abstract}
A volumetric error compensation method for a machining center that has multiple cutting tools operating simultaneously has been developed. Due to axis sharing, the geometric errors of multi-spindle, concurrent cutting processes are characterized by a significant coupling of error components in each cutting tool. As a result, it is not possible to achieve exact volumetric error compensation for all axes. To minimize the overall volumetric error in simultaneous cutting, a method to determine compensation amount using weighted least squares has been proposed. This method also allows tolerance distribution of machining accuracy for different surfaces of a workpiece. A geometric error model has been developed using an arch-type, multi-spindle machine tool, and the error compensation simulation results based on this model are presented. The simulation results demonstrated effectiveness of the proposed error compensation algorithm for use with multi-spindle simultaneous cutting applications.
\end{abstract}

Keywords Compensation - Geometric error - Machine tool . Multi spindle $\cdot$ Simultaneous cutting

\section{Introduction}

In the industry quest for faster production speeds, execution of more than a single cutting operation in an individual machine tool (referred to as simultaneous machining) has become a promising alternative to increase machine tool throughput. The

\section{K.-G. Ahn · Z.J. Pasek}

Engineering Research Center for Reconfigurable Manufacturing Systems, The University of Michigan,

Ann Arbor, MI, USA

\section{B.-K. Min (}

School of Mechanical Engineering,

Yonsei University,

Seoul, 120-749, South Korea

E-mail: bkmin@yonsei.ac.kr

Tel.: +82-2-21235813

Fax: $+82-2-3122159$ idea of simultaneous machining has been explored for long time. For example, automotive industry has used simultaneous drilling process since the early 1910s. [1]

The simultaneous machining can be implemented in several ways, for example, by means of multi-spindle drilling heads [2], simultaneous cutting with multiple tools in a single spindle lathe [3], and machining multiple features simultaneously with a multi-spindle machining center (mill-turn machines). The use of a multi-spindle drilling head is the most frequently deployed simultaneous cutting process in automotive industry today. Although the multi-spindle milling processes have not been frequently used due to difficulties in their setup and control, during the last few years the simultaneous machining gained more attention from manufacturing industry because of its economic benefits. As a result, a number of new designs of multi-spindle machining centers have been introduced by machine tool companies and universities such as reconfigurable multi-spindle machine tools proposed by Koren and his colleagues (see Fig. 1) [4-6]. This paper focuses on a special case of simultaneous milling process using more than one milling spindle, which is categorized as multi-spindle simultaneous cutting (MSSC) in this research.

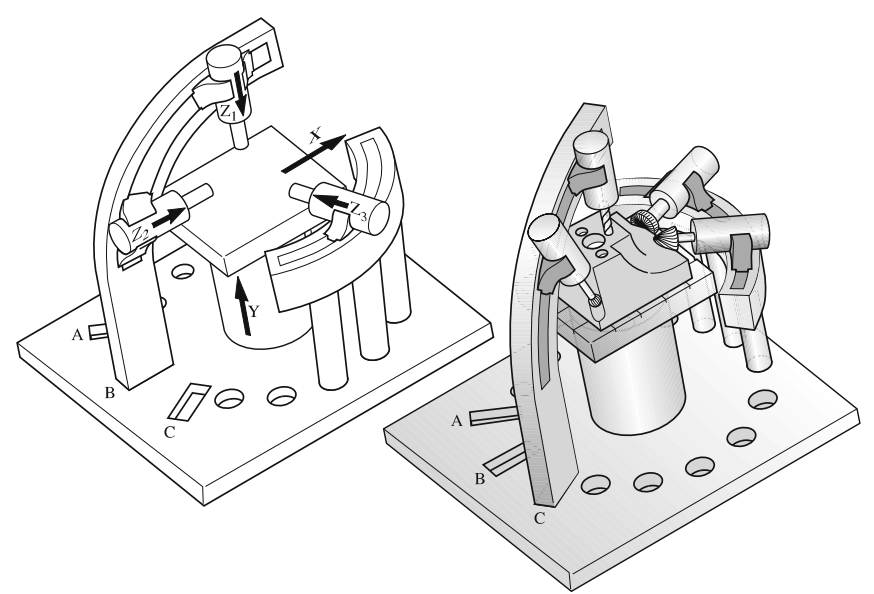

Fig. 1. Concept of reconfigurable multi-spindle machine tools [4] 
Besides its increased overall material removal ratio (MRR) due to the simultaneous use of multiple tools, the multi-spindle design in a single machine tool provides a range of benefits in productivity and machine tool cost. Obviously, addition of a spindle (and one or two additional axes if required) to the existing machine tool costs less than the addition of another machine tool to the production line to increase capacity. Tool change time and workpiece loading/unloading time can be kept at a minimum since the process requiring multiple cutting operations can be performed on a single machine tool without tool change and/or workpiece setup.

Multi-spindle machining centers currently available on the market are supposedly capable of the simultaneous machining of multiple features on a single workpiece. In practice however, multi-spindle characteristic are either utilized to process two to four identical workpieces in parallel or utilized to reduce the tool change time (e.g., tool change performed on one spindle, while the other one is machining).

For implementation of the MSSC on a single workpiece in actual production several issues must be considered. Analysis of dynamic characteristics, such as stability of the simultaneous machining process and impact of multiple cutting processes on the machining accuracy, is more difficult compared to that of single tool, single-spindle machining. Control, coordination, and error compensation of multiple spindle motion, especially when the motion of multiple spindles is coupled, also differ from those developed for conventional machine tools.

With increasing use of the multi-spindle machining centers in production $[7,8]$, some of these issues have been under investigation. Vibration problems of simultaneous cutting in turning were reported by Vorob'eva [9]. Stability of simultaneous machining using a single workpiece and multiple cutting tools in turning has been studied by Lazoglu et al. [10]. Ozdoganlar and Endres extended the mathematical stability analysis of simultaneous machining to twin turret turning machines and twin spindle turning machines [3].

Although more research results can be found in the topics such as, for example, process planning for multi-spindle machine tool [11] and reliability of multi-spindle machines [12], no substantial research has been conducted to investigate, model, and improve geometric accuracy and to implement it on the existing machine tool controllers. Because of varying degree of geometric coupling between error components in multiple cutting locations, it is impossible to achieve perfect compensation of all volumetric errors in MSSC.

This paper is focused on the modeling of geometric errors and the compensation of the errors for MSSC machining. The paper extends the machine tool geometric error modeling and error compensation theory developed for single spindle machine tools [13-18] to the MSSC case. A number of potential implementations of MSSC in milling processes are reviewed, followed by the error model synthesis for the MSSC. Furthermore, an error model for a recently-developed machine tool with archtype axis (aimed at automotive engine manufacturing to maximize the utilization of MSSC) is also introduced [5]. Because of the geometric coupling of axes in MSSC the error compensa- tion requires a new approach. This paper explains the limitations of error compensation for MSSC and proposes an approach to minimize geometric errors in MSSC.

Geometric problems of MSSC in various machine tool configurations are discussed in Sect. 2. Sect. 3 introduces the error model of a machine tool with an arch-type axis and a compensation method for MSSC. In Sect. 4 simulation results of geometric error compensation in MSSC are presented.

\section{Geometric problems in multi-spindle simultaneous cutting}

The merits of MSSC have been discussed in the previous section. The MSSC can be implemented in a number of different machine tool configurations. Each configuration for MSSC has its own, unique characteristics with regard to machining cost and geometric accuracy. In this section, a few examples of MSSC processes are presented and their aspects are reviewed to compare their characteristics.

The first MSSC example, where two identical products are made by dual-spindle MSSC is shown in Fig. 2a. The processes for two workpieces are identical and the two spindles are controlled by the same part program. Therefore, no special part program is required to control these two spindles simultaneously. This configuration can be used to reduce the manufacturing equipment cost when expanding its capacity - addition of an extra spindle into the existing machining center design is more economical than addition of a whole machine. Such a concept is often used in the recent designs of agile manufacturing lines. Because of relatively less acute dynamic problems appearing during the cutting process, this setup may be the most popular MSSC case that is currently used in production systems. However, the position of the multiple workpieces are determined by common axes, thus if there is a geometric error compensation required, this compensatory action for one workpiece may affect the position control of another workpiece. As a result, a problem of optimal geometric compensation may arise, an issue which will be discussed later in this paper.

Figure $2 b$ shows another example that the MSSC is used to process a single workpiece by using dual spindles on a common surface (used, for example, in aerospace industry for machining of large airframes on gantry machine tools). By introducing this
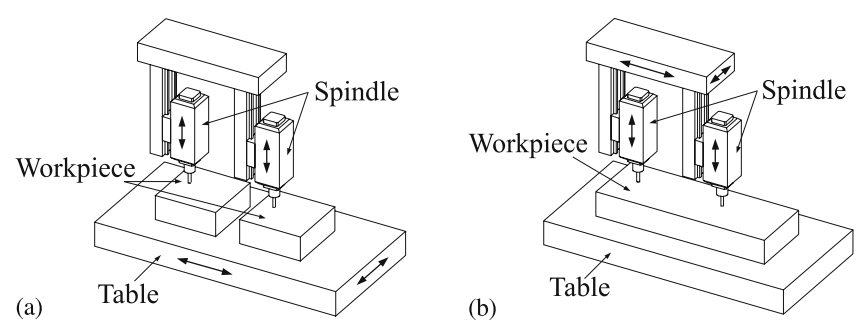

Fig. 2a,b. Geometry of multi-spindle simultaneous cutting a multiple workpiece with coupled table motion; b single workpiece with coupled spindle motion 
cutting scheme, the cycle time to produce a workpiece can be reduced by half when compared to a single-spindle machine tool. An additional benefit of this solution is that this machine is more failure tolerant in that if one spindle fails, the other spindle can still complete machining of the entire surface. Although it is not frequently used due to the aforementioned problems of simultaneous cutting, and this type of cutting process will be more popular in the near future as the dynamic problem is being alleviated and reconfigurable machining is being accepted in the industry. The geometric problem of this case is the same as that of Fig. 2a.

The design of MSSC introduced in Fig. $2 \mathrm{~b}$ can be generalized to maximize the benefits of MSSC. Figure 3 shows a multispindle RMT which is a good example of the cutting process in which motions are kinematically coupled by at least one axis motion. Geometric compensation problem from the axes shared by the spindles discussed above becomes more complicated in this configuration. While there may be a variety of designs and consequently a variety of resultant geometric couplings of the spindle motions, a typical case is shown in Fig. 3.

Sharing of the motion axes results in additional constraints in geometric error compensation problem. Consequently, the geometric error compensation prepared for one cutting tool error affects and may deteriorate the geometric accuracy of the process carried out by another cutting tool. If there is no sharing of motion axis between multiple spindles, such compound geometric problem does not exist. However, it is assumed that most

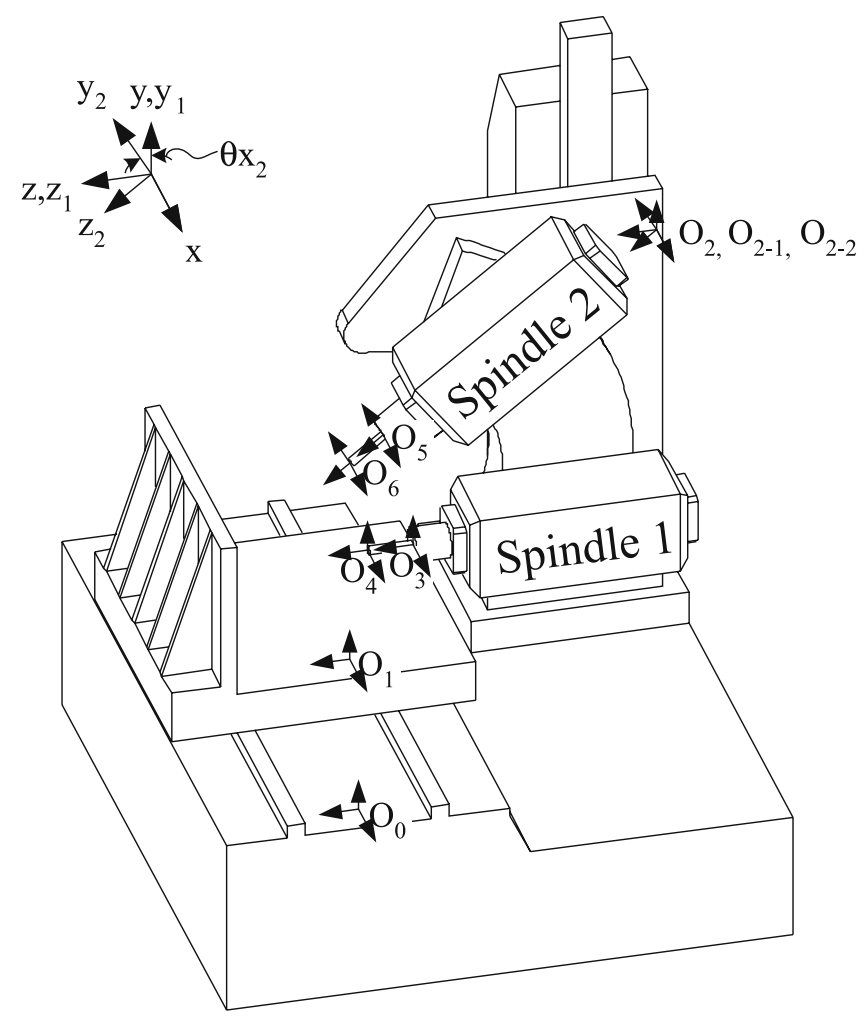

Fig. 3. The mechanical structure and the assigned coordinate systems of the multi-spindle RMT of MSSC belongs to either case in Figs. 2 or 3 because only when sharing of motion axis takes place, economic benefits of the MSSC can be claimed.

Many aspects of the proposed MSSC have been discussed in this and previous section. The new challenges identified, when compared to conventional machining, include, for example:

- Coupled geometric error in multiple spindles,

- Dynamic problems, and

- Selection of multiple cutting conditions for each of the multiple cutting tools.

From these issues, geometric error compensation for multiple spindles will be elaborated in detail in the remainder of this paper. A recently introduced reconfigurable machine tool (RMT) mentioned in Sect. 1 is considered as a possible application example of the geometric error compensation algorithm for MSSC, and is the focus of the next section.

\section{Volumetric error modeling and compensation for a multi-spindle machine tool}

As can be seen in Fig. 3, the two spindle axes are not parallel because the RMT was developed to process angled workpiece surfaces such as cylinder heads of V-type automotive engines. By enabling use of the cutting tools from different directions, by adjusting angular spindle location within a predefined range, the machine does not require the tilt axis, and the time required to reposition the tools or workpieces is greatly reduced. However, such configuration results in more complex motion planning to compensate the geometric errors, and the complexity of the coupled geometric errors increases with existence of multiple non-parallel spindles.

In this section, a model of the geometric errors in MSSC and a methodology to calculate the required compensation amount based on the kinematic solutions are developed. The conflicts of the compensation requirements between the coupled axes are demonstrated and an approach using accuracy weight factors for each machining feature is proposed. This scheme defines priority by which cutting accuracy using of each spindle can be managed within prescribed specifications. The results are presented in Sect. 4.

A three-dimensional error observed in a machine workspace as a function of the errors of machine members is derived. Rigid body kinematics and small angle approximations are employed to develop homogeneous transformations for inaccurate links and joints in the machine's kinematic chain as in the case for most machine tools. 21 geometric error components are used [19].

When a machine element is moving along a guideway of each axis, the errors in the axis scale result in the positional error. Generally, the positional error is the scale error plus the Abbe errors that arise from the Abbe offset and associated angular errors [19]. Hence the magnitude and direction of the positional error varies, depending on the location of each measurement point within the measurement volume (workspace). 
Three positional errors are considered in 3-axis machine tools. The out of straightness of the guideway gives the straightness error in the movement of the machine element that is moving on the guideway. The straightness errors are influenced by the associated rotational errors on the machine tools. There are two cases: the horizontal straightness error and the vertical straightness error, respectively, along each axis, and the six straightness errors are thus considered in a 3-axis machine tool. There are three rotational errors along a guideway. If the right-hand screw rule is adopted to describe the rotational movements, the feed direction determines the rotation axis. The roll error is associated with the rotation about the guideway, the pitch error is associated with the rotation about the horizontal transverse direction, and the yaw error is associated with the rotation about the vertical axis. It should be noted that these rotational errors contribute to the total volumetric error with the Abbe offset. Thus nine rotational errors are considered in a 3-axis machine tool. When the multi axis movement is introduced, the misalignment of each axis produces squareness errors (or orthogonality errors). In a 3-axis machine tool, three squareness errors are defined for the $X-Y, X-Z$, and $Y-Z$ planes. All the squareness errors are considered positive when they are outward from the right angle $\left(90^{\circ}\right)$. Therefore 18 error components plus three squareness errors fully describe error behavior of a 3-axis machine tool.

Multi-axis machines typically consist of one or more open kinematic chains composed of a sequence of elements or links connected by joints providing either a rotational or translational degree of freedom of motion. By successive application of the homogeneous transformation matrices of neighboring links in the kinematic chain of a machine tool, the position of a point in the last (tool) frame with respect to the first (reference) frame can be expressed. When errors exist, the actual coordinates are expressed as the variations of ${ }^{m-1} T_{m}$ :

${ }^{m-1} T_{m}^{a}={ }^{m-1} T_{m} \cdot E_{m}$

where the superscript $a$ refers to actual. The error of a kinematic joint from one coordinate to another can be expressed as:

$E_{m}=\left[\begin{array}{cccc}1 & -\varepsilon_{z} & \varepsilon_{y} & \delta_{x} \\ \varepsilon_{z} & 1 & -\varepsilon_{x} & \delta_{y} \\ -\varepsilon_{y} & \varepsilon_{x} & 1 & \delta_{z} \\ 0 & 0 & 0 & 1\end{array}\right]$

where $\varepsilon_{x}, \varepsilon_{y}$, and $\varepsilon_{z}$ are the angular errors associated with $x, y$, and $z$ axes, respectively, and $\delta_{x}, \delta_{y}$, and $\delta_{z}$ are the displacement errors due to imperfect geometry of machine tool axes.

The mechanical structure and the assigned coordinate systems of the multi-spindle machine tool in consideration are shown in Fig. 3. Spindle 1 mounted on the arc is movable along the $z_{1}$-axis slanted against the $z$-axis with $\theta_{x_{1}}$ degree, which is set at 0 degrees to make the model easy to understand. Spindle 2 mounted on the arc is movable along the $z_{2}$-axis slanted against the $z$-axis with $\theta_{x_{2}}$ degree. The movement of the workpiece along the $x$-axis is achieved by the movable slide mounted on the machine bed, and the movement of the tools along the $y$-axis (this motion involves the arch plate and both spindles) is achieved by the movable slide mounted on the machine column. There are nine coordinate frames in all, the reference, four movable slides, two tool tips, and two additional column coordinates expressing the locations of spindle 1 and spindle 2. Thus we have the origins of reference $\left(\mathrm{O}_{0}\right), x$-table slide $\left(\mathrm{O}_{1}\right)$, column $\left(\mathrm{O}_{2}\right)$, spindle $1\left(\mathrm{O}_{3}\right)$, tool tip of spindle $1\left(\mathrm{O}_{4}\right)$, spindle $2\left(\mathrm{O}_{5}\right)$, tool tip of spindle $2\left(\mathrm{O}_{6}\right)$, and two column coordinates $\left(\mathrm{O}_{2-1}, \mathrm{O}_{2-2}\right) . \mathrm{O}_{2}, \mathrm{O}_{2-1}$, and $\mathrm{O}_{2-2}$ hold one origin in common. $\mathrm{O}_{2-1}$ expresses the relation between $\mathrm{O}_{2}$ and $\mathrm{O}_{3}$, and $O_{2-2}$ expresses the relation between $O_{2}$ and $O_{5}$. The $x$ axis serves as the reference for the $x-y$ and the $x-z$ squareness errors, and the $y$-axis is the reference for the $y$ - $z$ squareness error.

The corresponding actual transformation matrices are listed below:

$\begin{aligned}{ }^{\mathbf{0}} \mathbf{T}_{\mathbf{1}}^{\mathbf{a}}= & {\left[\begin{array}{cccc}1 & -\varepsilon_{z x} & \varepsilon_{y x} & x+\delta_{x x} \\ \varepsilon_{z x} & 1 & -\varepsilon_{x x} & \delta_{y x} \\ -\varepsilon_{y x} & \varepsilon_{x x} & 1 & \delta_{z x} \\ 0 & 0 & 0 & 1\end{array}\right] } \\ { }^{\mathbf{0}} \mathbf{T}_{2}^{\mathbf{a}}= & {\left[\begin{array}{cccc}1 & -\varepsilon_{z y} & \varepsilon_{y y} & \delta_{x y}-S_{x y} y \\ \varepsilon_{z y} & 1 & -\varepsilon_{x y} & y+\delta_{y y} \\ -\varepsilon_{y y} & \varepsilon_{x y} & 1 & \delta_{z y} \\ 0 & 0 & 0 & 1\end{array}\right] }\end{aligned}$

${ }^{\mathbf{2}-1} \mathbf{T}_{\mathbf{3}}^{\mathbf{a}}=\left[\begin{array}{cccc}1 & -\varepsilon_{z_{1} z_{1}} & \varepsilon_{y_{1} z_{1}} & \delta_{x z_{1}}-S_{x z_{1}} z_{1} \\ \varepsilon_{z_{1} z_{1}} & 1 & -\varepsilon_{x z_{1}} & \delta_{y_{1} z_{1}}-S_{y_{1} z_{1}} z_{1} \\ -\varepsilon_{y_{1} z_{1}} & \varepsilon_{x z_{1}} & 1 & z_{1}+\delta_{z_{1} z_{1}} \\ 0 & 0 & 0 & 1\end{array}\right]$

${ }^{3} \mathbf{T}_{\mathbf{4}}=\left[\begin{array}{cccc}1 & 0 & 0 & 0 \\ 0 & 1 & 0 & 0 \\ 0 & 0 & 1 & Z_{4} \\ 0 & 0 & 0 & 1\end{array}\right]$

$\mathbf{T}_{\mathbf{w}-1}=\left[\begin{array}{cccc}1 & 0 & 0 & -x \\ 0 & 1 & 0 & y-\left(z_{1}+Z_{4}\right) \sin \theta x_{1} \\ 0 & 0 & 1 & \left(z_{1}+Z_{4}\right) \cos \theta x_{1} \\ 0 & 0 & 0 & 1\end{array}\right]$

$\mathbf{T}_{\mathbf{R X}}=\left[\begin{array}{cccc}1 & 0 & 0 & 0 \\ 0 & \cos \theta_{x_{1}} & -\sin \theta_{x_{1}} & 0 \\ 0 & \sin \theta_{x_{1}} & \cos \theta_{x_{1}} & 0 \\ 0 & 0 & 0 & 1\end{array}\right]$

$\mathbf{2}^{2} \mathbf{T}_{\mathbf{5}}^{\mathbf{a}}=\left[\begin{array}{cccc}1 & -\varepsilon_{z_{2} z_{2}} & \varepsilon_{y_{2} z_{2}} & \delta_{x z_{2}}-S_{x z_{2}} z_{2} \\ \varepsilon_{z_{2} z_{2}} & 1 & -\varepsilon_{x z_{2}} & \delta_{y_{2} z_{2}}-S_{y_{2} z_{2}} z_{2} \\ -\varepsilon_{y_{2} z_{2}} & \varepsilon_{x z_{2}} & 1 & z_{2}+\delta_{z_{2} z_{2}} \\ 0 & 0 & 0 & 1\end{array}\right]$

${ }^{5} \mathbf{T}_{\mathbf{6}}=\left[\begin{array}{cccc}1 & 0 & 0 & 0 \\ 0 & 1 & 0 & 0 \\ 0 & 0 & 1 & Z_{6} \\ 0 & 0 & 0 & 1\end{array}\right]$

$\mathbf{T}_{\mathbf{w}-2}=\left[\begin{array}{cccc}1 & 0 & 0 & -x \\ 0 & 1 & 0 & y-\left(z_{2}+Z_{6}\right) \sin \theta_{x} \\ 0 & 0 & 1 & \left(z_{2}+Z_{6}\right) \cos \theta_{x} \\ 0 & 0 & 0 & 1\end{array}\right]$ 
$\mathbf{T}_{\mathbf{R X}}=\left[\begin{array}{cccc}1 & 0 & 0 & 0 \\ 0 & \cos \theta_{x_{2}} & -\sin \theta_{x_{2}} & 0 \\ 0 & \sin \theta_{x_{2}} & \cos \theta_{x_{2}} & 0 \\ 0 & 0 & 0 & 1\end{array}\right]$

where ${ }^{0} \mathbf{T}_{1}^{\mathbf{a}}$ : the actual homogeneous transformation matrix (HTM) of the table slide in the reference frame. ${ }^{0} \mathbf{T}_{2}^{\mathbf{a}}$ : the actual HTM of the column in the reference frame. ${ }^{2-1} \mathbf{T}_{\mathbf{3}}^{\mathbf{a}}$ : the actual HTM of spindle 1 in the $O_{2-1}$ frame. ${ }^{3} \mathbf{T}_{\mathbf{4}}$ : the HTM of the cutting tool in the spindle 1 frame. ${ }^{2-2} \mathbf{T}_{\mathbf{5}}^{\mathrm{a}}$ : the actual HTM of spindle 2 in the $O_{2-2}$ frame. ${ }^{5} \mathbf{T}_{\mathbf{6}}$ : the HTM of the cutting tool in the spindle 2 frame. $\mathbf{T}_{\mathbf{w}-\mathbf{1}}$ : the HTM of the workpiece in the reference frame for spindle $1 . \mathbf{T}_{\mathbf{w}-2}$ : the HTM of the workpiece in the reference frame for spindle 2. $Z_{4}$ : the ideal tool dimension in the $z_{1}$ direction. $Z_{6}$ : the ideal tool dimension in the $z_{2}$ direction. $\mathbf{T}_{\mathbf{R X}}$ : the HTM that transforms the coordinates of a point in the $\mathrm{O}_{2-1}$ frame into the $\mathrm{O}_{2}$ frame. $\mathbf{T}_{\mathbf{R X}}$ : the HTM that transforms the coordinates of a point in the $O_{2-2}$ frame into the $O_{2}$ frame. $x, y, z, z_{1}, z_{2}$ : the joint displacements along the axes $x, y, z, z_{1}$, and $z_{2}$ respectively $. y_{1}, y_{2}$ : the axes obtained by having the $y$-axis rotate on the $x$-axis in $\theta_{x_{1}}$ and $\theta_{x_{2}}$ respectively. $\delta_{i j}$ : the positional error in the ith axis direction along jth axis $\left(i, j=x, y, z, y_{1}, z_{1}, y_{2}, z_{2}\right) . \varepsilon_{i j}$ : the angular errors, where the first subscript represents which axis the rotation error is around, and the second subscript represents the direction of movement of the slide ( $\left.i, j=x, y, z, y_{1}, z_{1}, y_{2}, z_{2}\right)$. $S_{x y_{1}}, S_{x z_{1}}, S_{y_{1} z_{1}}, S_{x z_{2}}, S_{y_{2} z_{2}}$ : squareness errors between the axis pairs.

Using these transformation matrices, the relative position and orientation errors between each tool and workpiece is represented by the following matrix multiplication:

$$
\begin{aligned}
& \mathbf{E}_{\text {total_1 }}=\left[{ }^{0} \mathbf{T}_{\mathbf{1}}^{\mathbf{a}} \mathbf{T}_{\mathbf{w} \_\mathbf{1}}\right]^{-1}\left[{ }^{0} \mathbf{T}_{2}^{\mathbf{a}}\right]\left[\mathbf{T}_{\mathbf{R}} \mathbf{x}_{1}\right]\left[\left[{ }^{2-1} \mathbf{T}_{3}^{\mathbf{a}}\right]\left[{ }^{3} \mathbf{T}_{4}\right]\right] \\
& \mathbf{E}_{\text {total_2 }}=\left[{ }^{0} \mathbf{T}_{1}^{\mathrm{a}} \mathbf{T}_{\mathbf{w}_{-} 2}\right]^{-1}\left[{ }^{0} \mathbf{T}_{\mathbf{2}}^{\mathrm{a}}\right]\left[\mathbf{T}_{\left.\mathbf{R} \mathbf{X}_{2}\right]}\right]\left[\left[{ }^{2-2} \mathbf{T}_{\mathbf{5}}^{\mathrm{a}}\right]\left[{ }^{5} \mathbf{T}_{\mathbf{6}}\right]\right] \text {. }
\end{aligned}
$$

After expanding the terms in Eqs. 13 and 14, the tool tip errors, $\mathbf{E}_{\text {total_1 }}$ and $\mathbf{E}_{\text {total_2 }}$ can be rewritten as Eqs. 15 and 16 .

$$
\begin{aligned}
\mathbf{E}_{\text {total } \_1} & =\left[\begin{array}{cccc}
e_{11} & e_{12} & e_{13} & P_{x_{1}} \\
e_{21} & e_{22} & e_{23} & P_{y_{1}} \\
e_{31} & e_{32} & e_{33} & P_{z_{1}} \\
0 & 0 & 0 & 1
\end{array}\right] \\
\mathbf{E}_{\text {total_2 }}= & {\left[\begin{array}{cccc}
e_{11} & e_{12} & e_{13} & P_{x_{2}} \\
e_{21} & e_{22} & e_{23} & P_{y_{2}} \\
e_{31} & e_{32} & e_{33} & P_{z_{2}} \\
0 & 0 & 0 & 1
\end{array}\right] }
\end{aligned}
$$

In general, if the amounts of errors are small, then only the first or the second order terms of approximation expansion can be used to model machine tool errors. Thus, in the derivation presented here, only the error terms up to the second order are considered. Using small angle assumption the positional error vectors of the two tool tips in the $x$-direction $\left(P_{x_{1}}\right.$ and $P_{x_{2}}$ in Eqs. 15 and 16, respectively) can be written as Eqs. 17 and 18.

$$
\begin{aligned}
& P_{x_{1}}=\left(\delta_{x x}-y \varepsilon_{z x}-\delta_{x y}-\delta_{x z_{1}}-Z_{4} \varepsilon_{y_{1} z_{1}}+y S_{x y}+z_{1} S_{x z_{1}}\right. \\
& +z_{1} \varepsilon_{y x} \cos \theta_{x_{1}}+Z_{4} \varepsilon_{y x} \cos \theta_{x_{1}}+z_{1} \varepsilon_{z x} \sin \theta_{x_{1}}+Z_{4} \varepsilon_{z x} \sin \theta_{x_{1}} \\
& -z_{1} \varepsilon_{y y} \cos \theta_{x_{1}}-Z_{4} \varepsilon_{y y} \cos \theta_{x_{1}}-z_{1} \varepsilon_{z y} \sin \theta_{x_{1}}-Z_{4} \varepsilon_{z y} \sin \theta_{x_{1}} \\
& +\varepsilon_{z x} \delta_{y x}-\varepsilon_{y x} \delta_{z x}-\varepsilon_{z x} \delta_{y y}+\varepsilon_{y x} \delta_{z y}+\varepsilon_{y x} \delta_{y_{1} z_{1}} \sin \theta_{x_{1}} \\
& -\varepsilon_{y y} \delta_{y_{1} z_{1}} \sin \theta_{x_{1}}-\varepsilon_{z x} \delta_{y_{1} z_{1}} \cos \theta_{x_{1}}+\varepsilon_{z y} \delta_{y_{1} z_{1}} \cos \theta_{x_{1}} \\
& +\varepsilon_{y x} \delta_{z_{1} z_{1}} \cos \theta_{x_{1}}+\varepsilon_{z x} \delta_{z_{1} z_{1}} \sin \theta_{x_{1}}-\varepsilon_{y y} \delta_{z_{1} z_{1}} \cos \theta_{x_{1}} \\
& -\varepsilon_{z y} \delta_{z_{1} z_{1}} \sin \theta_{x_{1}}-y \varepsilon_{y x} \varepsilon_{x x}-z_{1} \varepsilon_{z x} \varepsilon_{x x} \cos \theta_{x_{1}} \\
& -Z_{4} \varepsilon_{z x} \varepsilon_{x x} \cos \theta-x_{1}+z_{1} \varepsilon_{x x} \varepsilon_{y x} \sin \theta_{x_{1}}+Z_{4} \varepsilon_{x x} \varepsilon_{y x} \sin \theta_{x_{1}} \\
& -x \varepsilon_{y x}^{2}-x \varepsilon_{z x}^{2}-z_{1} \varepsilon_{y x} \varepsilon_{x y} \sin \theta_{x_{1}} \\
& -Z_{4} \varepsilon_{y x} \varepsilon_{x y} \sin \theta_{x_{1}}+z_{1} \varepsilon_{z x} \varepsilon_{x y} \cos \theta_{x_{1}}+Z_{4} \varepsilon_{z x} \varepsilon_{x y} \cos \theta_{x_{1}} \\
& -Z_{4} \varepsilon_{y x} \varepsilon_{x z_{1}} \sin \theta_{x_{1}}+Z_{4} \varepsilon_{y y} \varepsilon_{x z_{1}} \sin \theta_{x_{1}}-Z_{4} \varepsilon_{z y} \varepsilon_{x z_{1}} \cos \theta_{x_{1}} \\
& +Z_{4} \varepsilon_{z x} S_{x z_{1}} \cos \theta_{x_{1}}-z_{1} \varepsilon_{y x} S_{y_{1} z_{1}} \sin \theta_{x_{1}}+z_{1} \varepsilon_{z x} S_{y_{1} z_{1}} \cos \theta_{x_{1}} \\
& \left.+z_{1} \varepsilon_{y y} S_{y_{1} z_{1}} \sin \theta_{x_{1}}-z_{1} \varepsilon_{z y} S_{y_{1} z_{1}} \cos \theta_{x_{1}}\right) \\
& /\left(1+\varepsilon_{x x}^{2}+\varepsilon_{y x}^{2}+\varepsilon_{z x}^{2}\right) \\
& P_{x_{2}}=\left(\delta_{x x}-y \varepsilon_{z x}-\delta_{x y}-\delta_{x z_{2}}-Z_{6} \varepsilon_{y_{2} z_{2}}+y S_{x y}+z_{2} S_{x z_{2}}\right. \\
& +z_{2} \varepsilon_{y x} \cos \theta_{x_{2}}+Z_{6} \varepsilon_{y x} \cos \theta_{x_{2}}+z_{2} \varepsilon_{z x} \sin \theta_{x_{2}} \\
& +Z_{6} \varepsilon_{z x} \sin \theta_{x_{2}}-z_{2} \varepsilon_{y y} \cos \theta_{x_{2}}-Z_{6} \varepsilon_{y y} \cos \theta_{x_{2}} \\
& -z_{2} \varepsilon_{z y} \sin \theta_{x_{2}}-Z_{6} \varepsilon_{z y} \sin \theta_{x_{2}}+\varepsilon_{z x} \delta_{y x}-\varepsilon_{y x} \delta_{z x} \\
& -\varepsilon_{z x} \delta_{y y}+\varepsilon_{y x} \delta_{z y}+\varepsilon_{y x} \delta_{y_{2} z_{2}} \sin \theta_{x_{2}}-\varepsilon_{y y} \delta_{y_{2} z_{2}} \sin \theta_{x_{2}} \\
& -\varepsilon_{z x} \delta_{y_{2} z_{2}} \cos \theta_{x_{2}}+\varepsilon_{z y} \delta_{y_{2} z_{2}} \cos \theta_{x_{2}}+\varepsilon_{y x} \delta_{z_{2} z_{2}} \cos \theta_{x_{2}} \\
& +\varepsilon_{z x} \delta_{z_{2} z_{2}} \sin \theta_{x_{2}}-\varepsilon_{y y} \delta_{z_{2} z_{2}} \cos \theta_{x_{2}}-\varepsilon_{z y} \delta_{z_{2} z_{2}} \sin \theta_{x 2} \\
& -y \varepsilon_{y x} \varepsilon_{x x}-z_{2} \varepsilon_{z x} \varepsilon_{x x} \cos \theta_{x_{2}}-Z_{6} \varepsilon_{z x} \varepsilon_{x x} \cos \theta_{x_{2}} \\
& +z_{2} \varepsilon_{x x} \varepsilon_{y x} \sin \theta_{x_{2}}+Z_{6} \varepsilon_{x x} \varepsilon_{y x} \sin \theta_{x_{2}}-x \varepsilon_{y x}^{2}-x \varepsilon_{z x}^{2} \\
& -z_{2} \varepsilon_{y x} \varepsilon_{x y} \sin \theta_{x_{2}}-Z_{6} \varepsilon_{y x} \varepsilon_{x y} \sin \theta_{x_{2}}+z_{2} \varepsilon_{z x} \varepsilon_{x y} \cos \theta_{x_{2}} \\
& +Z_{6} \varepsilon_{z x} \varepsilon_{x y} \cos \theta_{x_{2}}-Z_{6} \varepsilon_{y x} \varepsilon_{x z_{2}} \sin \theta_{x_{2}}+Z_{6} \varepsilon_{y y} \varepsilon_{x z_{2}} \sin \theta_{x_{2}} \\
& -Z_{6} \varepsilon_{z y} \varepsilon_{x z_{2}} \cos \theta_{x_{2}}+Z_{6} \varepsilon_{z x} S_{x z_{2}} \cos \theta_{x_{2}}-z_{2} \varepsilon_{y x} S_{y_{2} z_{2}} \sin \theta_{x_{2}} \\
& \left.+z_{2} \varepsilon_{z x} S_{y_{2} z_{2}} \cos \theta_{x_{2}}+z_{2} \varepsilon_{y y} S_{y_{2} z_{2}} \sin \theta_{x_{2}}-z_{2} \varepsilon_{z y} S_{y_{2} z_{2}} \cos \theta_{x_{2}}\right) \\
& /\left(1+\varepsilon_{x x}^{2}+\varepsilon_{y x}^{2}+\varepsilon_{z x}^{2}\right)
\end{aligned}
$$

Similarly, the positional error vectors in $y$ - and $z$-directions $\left(P_{y_{1}}\right.$, $P_{z_{1}}, P_{y_{2}}, P_{z_{2}}$ ) can be obtained and are given in Appendix A.

The positional error vectors in Eqs. 15 and 16 can be combined into a composite positional error vector as Eq. 19. The positional error compensation vector in the reference coordinate frame $\left(\mathbf{C}_{\text {ref }}^{\mathbf{T}}\right)$ is obtained in Eq. 20.

$$
\begin{aligned}
& \mathbf{P}^{\mathbf{T}}=\left[\begin{array}{llllll}
P_{x_{1}} & P_{y_{1}} & P_{z_{1}} & P_{x_{2}} & P_{y_{2}} & P_{z_{2}}
\end{array}\right] \\
& \mathbf{C}_{\mathrm{ref}}^{\mathbf{T}}=-\mathbf{P}^{\mathbf{T}}=\left[\begin{array}{llllll}
C_{r x_{1}} & C_{r y_{1}} & C_{r z_{1}} & C_{r x_{2}} & C_{r y_{2}} & C_{r z_{2}}
\end{array}\right]
\end{aligned}
$$

To enable implementation of the compensation method in the actual machine control, the compensation vector has to be converted from the reference frame to the frames describing the motion of the actuators. The number of components in the positional error compensation vector is reduced from six to four due to the geometric coupling as shown in Eq. 21.

$\mathbf{C}_{\mathrm{act}}^{\mathbf{T}}=\left[\begin{array}{llll}C_{a x} & C_{a y} & C_{a z_{1}} & C_{a z_{2}}\end{array}\right]$ 
Equation 21 is the positional error compensation vector in the actuator frames $\left(\mathbf{C}_{\mathrm{act}}\right)$. Eqs. 22 and 23 show the relation between $\mathbf{C}_{\text {ref }}$ and $\mathbf{C}_{\text {act }}$ as well as the corresponding transformation matrix.

$$
\begin{aligned}
\mathbf{C}_{\mathrm{ref}}=\left[{ }_{\mathrm{ref}} \mathbf{T}_{\left.\mathbf{C}_{\mathrm{act}}\right]\left[\mathbf{C}_{\mathrm{act}}\right]}\right. \\
\mathbf{C}_{\mathrm{ref}} \mathbf{T}_{\mathbf{C}_{\mathrm{act}}}=\left[\begin{array}{cccc}
1 & 0 & 0 & 0 \\
0 & 1 & -\sin \theta_{x_{1}} & 0 \\
0 & 0 & \cos \theta_{x_{1}} & 0 \\
1 & 0 & 0 & 0 \\
0 & 1 & 0 & -\sin \theta_{x_{2}} \\
0 & 0 & 0 & \cos \theta_{x_{2}}
\end{array}\right]
\end{aligned}
$$

$\mathbf{C}_{\text {ref }} \mathbf{T}_{\mathbf{C}_{\text {act }}}$ is non-symmetric because of the coupling of the axes. To obtain $\mathbf{C}_{\text {act }}$ the weighted least squares method is applied. Each element of $\mathbf{C}_{\text {act }}$ is written as Eqs. 24a to 28a.

$$
\mathbf{C}_{\mathrm{act}}=\left[\left[\mathbf{C}_{\mathrm{ref}} \mathbf{T}_{\mathbf{C}_{\mathrm{act}}}\right]^{T} \mathbf{W}\left[{ }^{\mathbf{C}_{\mathrm{ref}}} \mathbf{T}_{\mathbf{C}_{\mathrm{act}}}\right]\right]^{-1}\left[\left[\mathbf{C}_{\mathrm{ref}} \mathbf{T}_{\mathbf{C}_{\mathrm{act}}}\right]^{T} \mathbf{W} \mathbf{C}_{\mathrm{ref}}\right]
$$$$
\mathbf{W}=\left[\begin{array}{cccccc}
w_{1} & 0 & 0 & 0 & 0 & 0 \\
0 & w_{2} & 0 & 0 & 0 & 0 \\
0 & 0 & w_{3} & 0 & 0 & 0 \\
0 & 0 & 0 & w_{4} & 0 & 0 \\
0 & 0 & 0 & 0 & w_{5} & 0 \\
0 & 0 & 0 & 0 & 0 & w_{6}
\end{array}\right]
$$

$C_{a x}=\frac{w_{1} C_{r x_{1}}+w_{4} C_{r x_{2}}}{w_{1}+w_{4}}$

$C_{a y}=\frac{N C_{a y}}{D C_{a y}}$

$N C_{a y}=w_{2} w_{3} \sin ^{2} \theta_{x_{1}} \cos ^{2} \theta_{x_{1}}\left(w_{5} \sin ^{2} \theta_{x_{2}}+w_{6} \cos ^{2} \theta_{x_{2}}\right)$

$\times\left(w_{2} C_{r y_{1}}+w_{5} C_{r y_{2}}\right)+w_{2} \sin \theta_{x_{1}}\left(w_{5} \sin ^{2} \theta_{x_{2}}+w_{6} \cos ^{2} \theta_{x_{2}}\right)$

$\times\left(-w_{2} C_{r y_{1}} \sin \theta_{x_{1}}+w_{3} C_{r z_{1}} \cos ^{2} \theta_{x_{1}}\right)+w_{5} \sin \theta_{x_{2}}$

$\times\left(w_{2} \sin ^{2} \theta_{x_{1}}+w_{3} \cos ^{2} \theta_{x_{1}}\right)\left(-w_{5} C_{r y_{2}} \sin \theta_{x_{2}}+w_{6} C_{r z_{2}} \cos \theta_{x_{2}}\right)$

$D C_{a y}=w_{2} w_{3} w_{5} \cos ^{2} \theta_{x_{1}} \sin ^{2} \theta_{x_{2}}+w_{2} w_{3} w_{6} \cos ^{2} \theta_{x_{1}} \cos ^{2} \theta_{x_{2}}$ $+w_{2} w_{5} w_{6} \sin ^{2} \theta_{x_{1}} \cos ^{2} \theta_{x_{2}}+w_{3} w_{5} w_{6} \cos ^{2} \theta_{x_{1}} \cos ^{2} \theta_{x_{2}}$

$C_{a z_{1}}=\frac{N C_{a z_{1}}}{D C_{a z_{1}}}$

$N C_{a z_{1}}=w_{2} \sin \theta_{x_{1}}\left(w_{5} \sin ^{2} \theta_{x_{2}}+w_{6} \cos ^{2} \theta_{x_{2}}\right)$ $\times\left(w_{2} C_{r y_{1}}+w_{5} C_{r y_{2}}\right)$

$+\left(w_{2} w_{5} \sin ^{2} \theta_{x_{2}}+w_{2} w_{6} \cos ^{2} \theta_{x_{2}}+w_{5} w_{6} \cos ^{2} \theta_{x_{2}}\right)$

$\times\left(-w_{2} C_{r y_{1}} \sin \theta_{x_{1}}+w_{3} C_{r z_{1}} \cos \theta_{x_{1}}\right)+w_{2} w_{5} \sin \theta_{x_{1}} \sin \theta_{x_{2}}$

$\times\left(-w_{5} C_{r y_{2}} \sin \theta_{x_{2}}+w_{6} C_{r z_{2}} \cos \theta_{x_{2}}\right)$

$D C_{a z_{1}}=w_{2} w_{3} w_{5} \cos ^{2} \theta_{x_{1}} \sin ^{2} \theta_{x_{2}}+w_{2} w_{3} w_{6} \cos ^{2} \theta_{x_{1}} \cos ^{2} \theta_{x_{2}}$ $+w_{2} w_{5} w_{6} \sin ^{2} \theta_{x_{1}} \cos ^{2} \theta_{x_{2}}+w_{3} w_{5} w_{6} \cos ^{2} \theta_{x_{1}} \cos ^{2} \theta_{x_{2}}$

$$
C_{a z_{2}}=\frac{N C_{a z_{2}}}{D C_{a z_{2}}}
$$

$N C_{a z_{2}}=w_{5} \sin \theta_{x_{2}}\left(w_{2} \sin ^{2} \theta_{x_{1}}+w_{3} \cos ^{2} \theta_{x_{1}}\right)$

$$
\times\left(w_{2} C_{r y_{1}}+w_{5} C_{r y_{2}}\right)
$$

$+w_{2} w_{5} \sin \theta_{x_{1}} \sin \theta_{x_{2}}\left(-w_{2} C_{r y_{1}} \sin \theta_{x_{1}}+w_{3} C_{r z_{1}} \cos \theta_{x_{1}}\right)$

$+\left(w_{2} w_{3} \cos ^{2} \theta_{x_{1}}+w_{2} w_{5} \sin ^{2} \theta_{x_{1}}+w_{3} w_{5} \cos ^{2} \theta_{x_{1}}\right)$

$\times\left(-w_{5} C_{r y_{2}} \sin \theta_{x_{2}}+w_{6} C_{r z_{2}} \cos \theta_{x_{2}}\right)$

$D C_{a z_{2}}=w_{2} w_{3} w_{5} \cos ^{2} \theta_{x_{1}} \sin ^{2} \theta_{x_{2}}+w_{2} w_{3} w_{6} \cos ^{2} \theta_{x_{1}} \cos ^{2} \theta_{x_{2}}$

$+w_{2} w_{5} w_{6} \sin ^{2} \theta_{x_{1}} \cos ^{2} \theta_{x_{2}}+w_{3} w_{5} w_{6} \cos ^{2} \theta_{x_{1}} \cos ^{2} \theta_{x_{2}}$

where P: the positional error vector in the reference frame. $\mathbf{C}_{\text {ref }}$ : the positional error compensation vector in the reference frame. $C_{r x_{1}}, C_{r y_{1}}, C_{r z_{1}}$ : the elements of the positional error compensation vector for spindle 1 in the reference frame in the $\mathrm{x}, \mathrm{y}$, and $\mathrm{z}$ directions, respectively. $C_{r x_{2}}, C_{r y_{2}}, C_{r z_{2}}$ : the elements of the positional error compensation vector for spindle 2 in the reference frame in the $\mathrm{x}, \mathrm{y}$, and $\mathrm{z}$ directions, respectively. $\mathbf{C}_{\text {act }}$ : the positional error compensation vector in the actuators' frames. $C_{a x}, C_{a y}, C_{a z_{1}}, C_{a z_{2}}$ : the elements of the positional error compensation vector in the actuators' frames in the $x, y$, $z_{1}$, and $z_{2}$ directions, respectively. $\mathbf{C}_{\mathrm{ref}} \mathbf{T}_{\mathbf{C}_{\text {act }}}$ : the transformation matrix that transforms the coordinates of a point in the actuators' frames into the reference frame. W: the weight matrix. $w_{1}, w_{2}, w_{3}, w_{4}, w_{5}, w_{6}$ : the diagonal elements of the weight matrix.

The weighted least square method incorporates nonnegative weights into the fitting criterion. The value of the weight indicates the precision of the information contained in the associated data. In the proposed method this feature is utilized to minimize the overall volumetric error or to determine the compensation amount for each spindle axis in simultaneous cutting, which allows tolerance distribution of machining accuracy for different surfaces of a workpiece. By adjusting the weight factors in Eq. 24b the relative tolerance among machining directions of both spindles can be determined. The proposed scheme utilizing weighted least square can be generally applied to various configurations of multispindle tools, such as Figs. 2a and b, to obtain the compensation vector as well as tolerance distribution. The simulation results of the multi-spindle machine tool volumetric error compensation are presented and discussed in the next section.

\section{Simulation results}

Because the multi-spindle RMT is in a conceptual design stage, the results presented in this section were obtained by simulation. To enhance the reality of the simulation, the machine tool error models were obtained by experiment using a real vertical-type three-axis machine tool with 510, 300, and $310 \mathrm{~mm}$ feed strokes in the $x$-, $y$-, and $z$-axis directions, respectively. The $z$-axis data set was used twice to model the two spindles of the multi-spindle RMT. The measured workspace range of the machine tool is as follows: $x$-axis: 10 to $500 \mathrm{~mm}$; $y$-axis: 10 to $290 \mathrm{~mm} ; z_{1}$ - and $z_{2}$ axis: -300 to $-10 \mathrm{~mm}$. The volumetric error in any location in the workspace can be derived using the proposed model. 
The simulation results are summarized in Tables 1 and 2 . Table 1 presents the effect of geometric coupling and the Table 2 demonstrates the performance of the proposed compensation method.

Table 1 shows the maximum residual volumetric errors of each spindle before the compensation was made, and compares the volumetric errors of spindle 1 and 2 when the conventional compensations were made only for a single axis. As can be seen in the table, when the compensation was applied to only spindle 1 although the residual volumetric error of spindle 1 was reduced below $1 \mu \mathrm{m}$, the volumetric error of spindle 2 re-

Table 1. Maximum residual errors in the workspace (unit: $\mu \mathrm{m}$ )

\begin{tabular}{|c|c|c|c|c|}
\hline & \multirow[t]{2}{*}{ Direction } & \multirow{2}{*}{$\begin{array}{l}\text { Max. residual } \\
\text { error before } \\
\text { compensation }\end{array}$} & \multicolumn{2}{|c|}{$\begin{array}{l}\text { Max. residual error } \\
\text { after Compensation }\end{array}$} \\
\hline & & & Spindle 1 & Spindle 2 \\
\hline \multirow[t]{3}{*}{ Spindle 1} & $\mathrm{x}$ & 193.55 & 0.99 & 32.82 \\
\hline & $\mathrm{y}$ & 161.09 & 0.99 & 82.48 \\
\hline & Z & 116.28 & 0.99 & 42.91 \\
\hline \multirow[t]{3}{*}{ Spindle 2} & $\mathrm{x}$ & 186.89 & -32.76 & 0.99 \\
\hline & $\mathrm{y}$ & 160.35 & -82.48 & 0.99 \\
\hline & $\mathrm{Z}$ & 84.26 & -42.90 & 0.99 \\
\hline
\end{tabular}

mained above $30 \mu \mathrm{m}$ in all directions. When the compensation was made for spindle 2 only, the machine tool error exhibited a similar trend. The result demonstrates how the geometric coupling between axes influences the accuracy of the geometric error compensation of MSSC machining. As can be readily noticed, for MSSC machining the volumetric errors of all tool tip positions cannot be simultaneously reduced to near zero. Therefore, a new compensation strategy is required to determine compensation amount for each spindle axis properly in the simultaneous cutting.

Table 2 shows the maximum residual volumetric errors of each spindle with respect to the variation of the weighting parameters when the proposed compensation method was applied. Three different combinations of the weights were applied to the compensation algorithm. Case 1 used equal weighting factors for all directions of both spindles (i.e., $w_{1}=w_{2}=\ldots=w_{6}=1$ ), case 2 used ten times bigger weighting factors for spindle 1 (i.e., $w_{1}=w_{2}=w_{3}=10$ and $w_{4}=w_{5}=w_{6}=1$ ), and case 3 used ten times bigger weighting factors for spindle 2 (i.e., $w_{1}=w_{2}=w_{3}=1$ and $\left.w_{4}=w_{5}=w_{6}=10\right)$.

To better evaluate the simulation results using the proposed method part of the results are also graphically presented in Figs. 4 to 9. The graphs show the volumetric errors after compensation in the three directions $(x-, y$-, and $z$-directions)
Table 2. Maximum residual errors according to the weighting parameters (unit: $\mu \mathrm{m}$ )

\begin{tabular}{|c|c|c|c|c|c|c|c|c|}
\hline & \multicolumn{2}{|c|}{ Weighting parameters } & \multicolumn{2}{|c|}{$\begin{array}{l}\text { Max. residual } \\
\text { error in x-direction }\end{array}$} & \multicolumn{2}{|c|}{$\begin{array}{l}\text { Max. residual } \\
\text { error in y-direction }\end{array}$} & \multicolumn{2}{|c|}{$\begin{array}{l}\text { Max. residual } \\
\text { error in } \mathrm{z} \text {-direction }\end{array}$} \\
\hline & $w_{1}, w_{2}, w_{3}$ & $w_{4}, w_{5}, w_{6}$ & Spindle1 & Spindle2 & Spindle 1 & Spindle 2 & Spindle 1 & Spindle 2 \\
\hline Case 1 & 1 & 1 & 16.43 & -16.37 & 7.37 & -7.35 & 0.99 & -7.36 \\
\hline Case 2 & 10 & 1 & 3.23 & -29.79 & 1.81 & -10.50 & 0.99 & -10.51 \\
\hline Case 3 & 1 & 10 & 29.85 & -3.34 & 18.41 & -2.30 & 0.99 & -2.38 \\
\hline
\end{tabular}

Fig. 4a-c. Residual volumetric error maps for spindle 1 after compensation (in case 1) a on $y-z$ plane $(x=250 \mathrm{~mm}) ; \mathbf{b}$ on $\mathrm{x}-\mathrm{z}$ plane $(y=150 \mathrm{~mm}) ; \mathbf{c}$ on $\mathrm{x}-\mathrm{y}$ plane $(z=-150 \mathrm{~mm})$

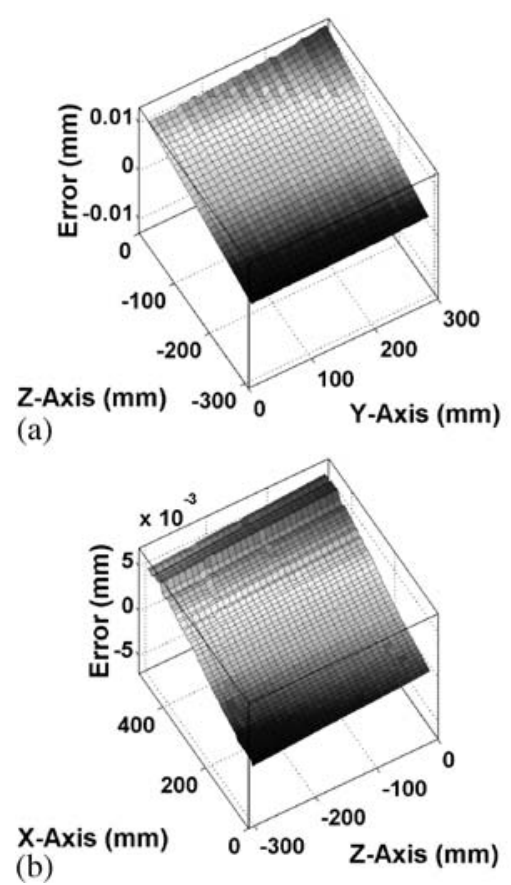

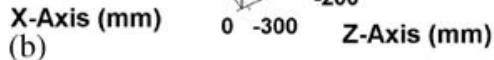

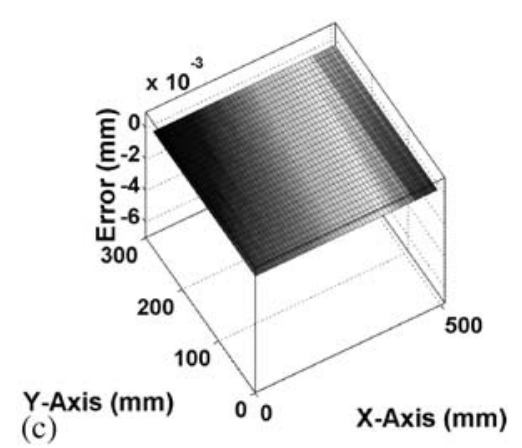


Fig. 5a-c. Residual volumetric error maps for spindle 2 after compensation (in case 1) a on y-z plane $(x=250 \mathrm{~mm}) ; \mathbf{b}$ on $\mathrm{x}-\mathrm{z}$ plane $(y=150 \mathrm{~mm}) ; \mathbf{c}$ on $\mathrm{x}-\mathrm{y}$ plane $(z=-150 \mathrm{~mm})$

Fig. 6a-c. Residual volumetric error maps for spindle 1 after compensation (in case 2 ) a on $y$-z plane $(x=250 \mathrm{~mm}) ; \mathbf{b}$ on $\mathrm{x}-\mathrm{z}$ plane $(y=150 \mathrm{~mm}) ; \mathbf{c}$ on $\mathrm{x}-\mathrm{y}$ plane $(z=-150 \mathrm{~mm})$

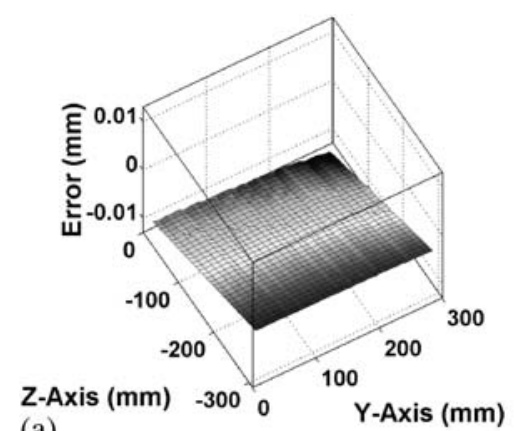

(a)

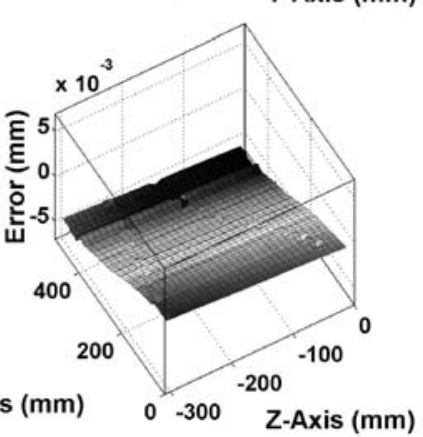

(b)

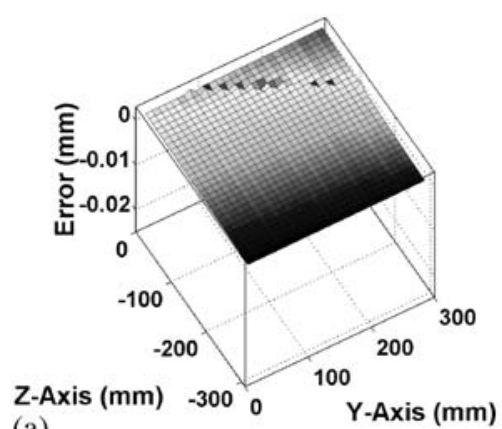

(a)

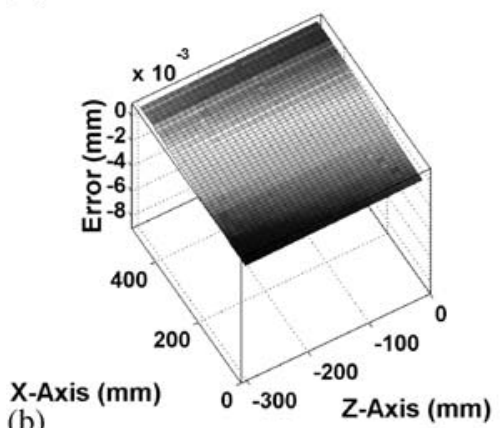

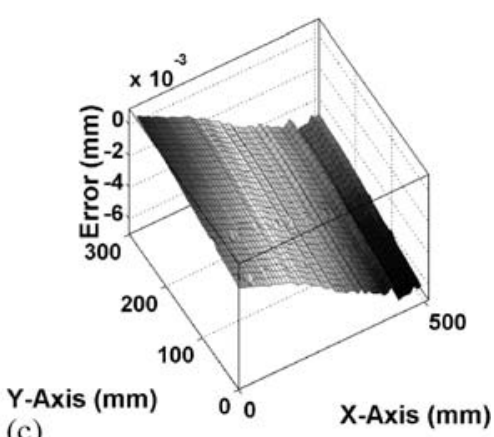

(c)

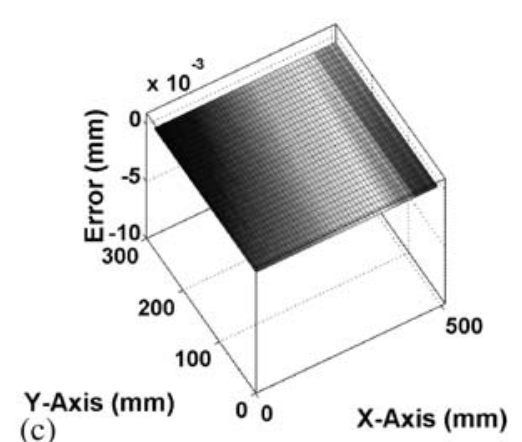

in the machine tool workspaces. Figures 4 and 5 show the residual errors after compensation of case 1 of Table 2. Figure $4 \mathrm{a}$ shows the residual error of spindle 1 in the $x$-direction along the $y$ - and $z$-axes when the nominal position of the $x$ axis is $250 \mathrm{~mm}$ and Fig. $4 \mathrm{~b}$ and $\mathrm{c}$ in the $x$ - and $z$-directions. In the same way, Fig. 5 shows the residual error of spindle 2. Figures 6 and 7 show the residual errors in case 2, and Figs. 8 and 9 show the residual errors in case 3 in the same way.
As can be seen in the table and graphs, the proposed method reduced the position error of the both spindles to less than $17 \mu \mathrm{m}$ during the simultaneous cutting when the equal weighting factors were used. Furthermore, it was possible to manage the tolerance distribution of machining accuracy for different surfaces of a workpiece. For example, in case 2, the position error of spindle 1 could be reduced within $4 \mu \mathrm{m}$ while the error of the other spindle was maintained less than $30 \mu \mathrm{m}$, which was a significant improvement compared to the result displayed in Table 1. 
Fig. 7a-c. Residual volumetric error maps for spindle 2 after compensation (in case 2) a on y-z plane $(x=250 \mathrm{~mm}) ; \mathbf{b}$ on $\mathrm{x}-\mathrm{z}$ plane $(y=150 \mathrm{~mm}) ; \mathbf{c}$ on $\mathrm{x}-\mathrm{y}$ plane $(z=-150 \mathrm{~mm})$

Fig. 8a-c. Residual volumetric error maps for spindle 1 after compensation (in case 3 ) a on $\mathrm{y}$-z plane $(x=250 \mathrm{~mm}) ; \mathbf{b}$ on $\mathrm{x}-\mathrm{z}$ plane $(y=150 \mathrm{~mm}) ; \mathbf{c}$ on $\mathrm{x}-\mathrm{y}$ plane $(z=-150 \mathrm{~mm})$

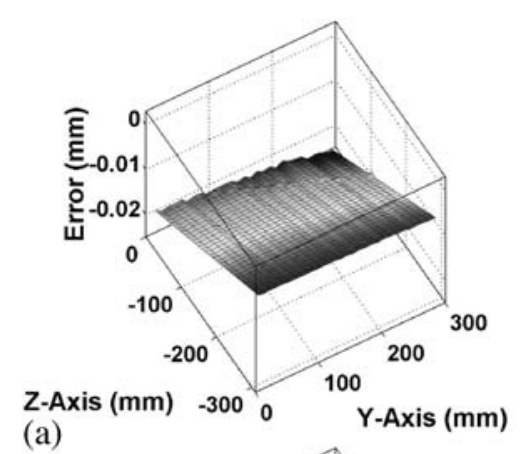

(a)

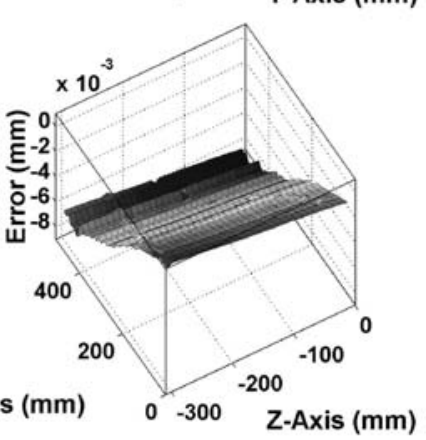

(b)

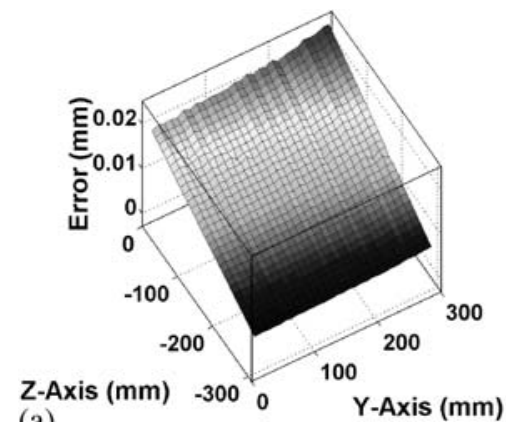

(a)

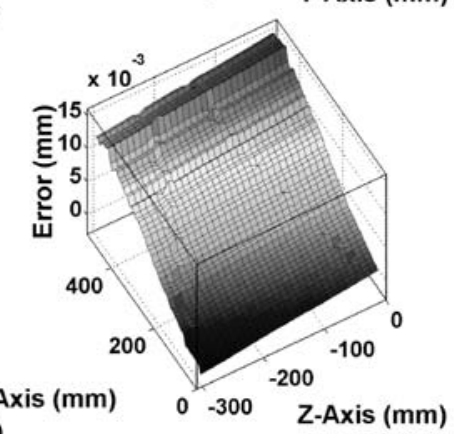

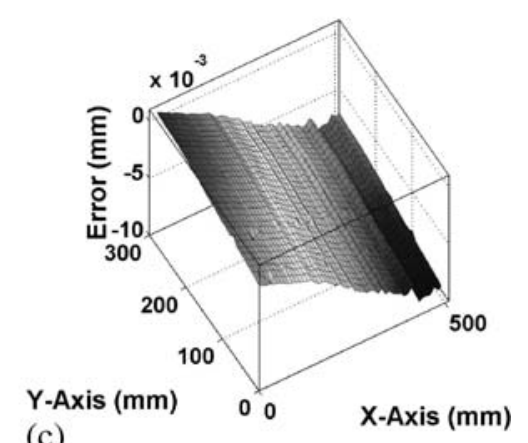

(c)

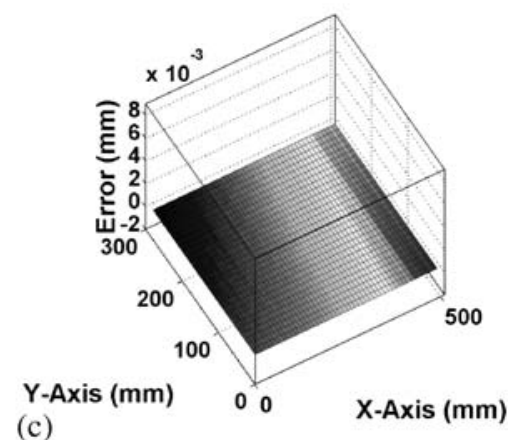

(c) (b)

\section{Conclusions}

In this paper the volumetric errors of multi-spindle machine tools were introduced, discussed and the corresponding error model has been presented. Based on the developed model, a new error compensation scheme for the geometrically-coupled multiple spindles has been developed. The proposed volumetric error compensation method in MSSC has been assessed by simulation study of an arch type multi-spindle machine tool. As demonstrated in Figs. 4 through 9 the simulation results confirm that the proposed methodology to compensate geometric errors in MSSC based on the weighted least squares method worked effectively in terms of error compensation as well as compensation conflict management. The maximum error of $194 \mu \mathrm{m}$ before compensation was reduced to maximum $17 \mu \mathrm{m}$ for all directions. By adjusting the weight factor introduced in the proposed method, the volumetric error of the target cutting feature could be reduced 
Fig. 9a-c. Residual volumetric error maps for spindle 2 after compensation (in case 3) a on y-z plane $(x=250 \mathrm{~mm}) ; \mathbf{b}$ on $\mathrm{x}-\mathrm{z}$ plane $(y=150 \mathrm{~mm}) ; \mathbf{c}$ on $\mathrm{x}-\mathrm{y}$ plane $(z=-150 \mathrm{~mm})$

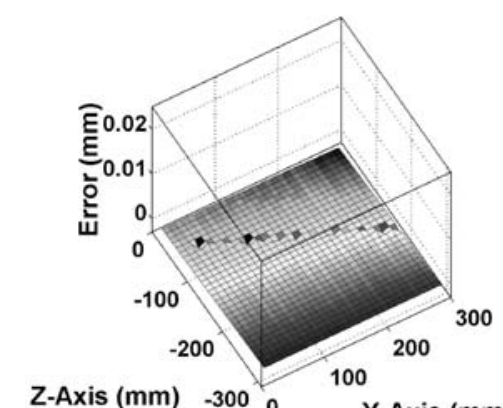

(a)

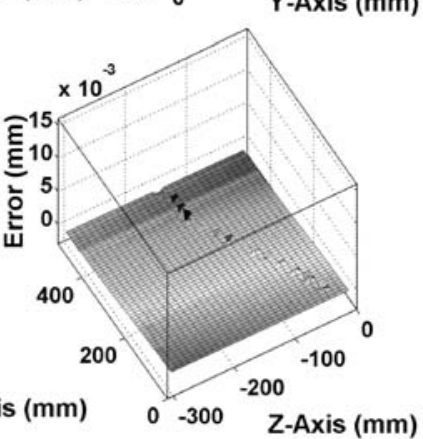

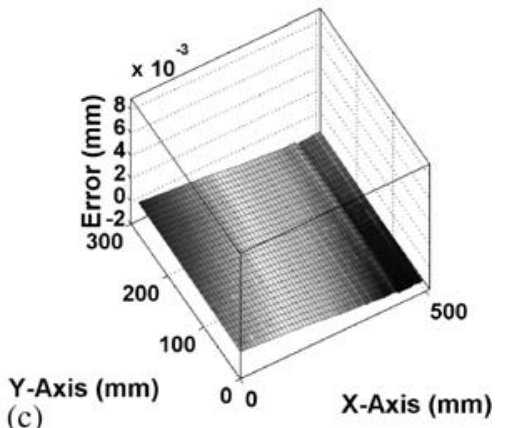

(b)

to $4 \mu \mathrm{m}$ and the ratio between the errors of two spindles could be adjusted while the accuracy of the other axis can be maintained within the desired value.

\section{Appendix A}

The positional error vectors of the multi spindles in $y$ - and $z$ directions $\left(P_{y_{1}}, P_{z_{1}}, P_{y_{2}}, P_{z_{2}}\right)$ are as follows.

$$
\begin{aligned}
& P_{y_{1}}=\left(\delta_{y x}+z_{1} y \varepsilon_{x x} \cos \theta_{x_{1}}+Z_{4} \varepsilon_{x x} \cos \theta_{x_{1}}+x \varepsilon_{z x}-z_{1} \varepsilon_{x y} \cos \theta_{x_{1}}\right. \\
& -Z_{4} \varepsilon_{x y} \cos \theta_{x_{1}}+\delta_{y y}-Z_{4} \varepsilon_{x z_{1}} \cos \theta_{x_{1}}+\delta_{y_{1} z_{1}} \cos \theta_{x_{1}} \\
& -\delta_{z_{1} z_{1}} \sin \theta_{x_{1}}-z_{1} S_{y_{1} z_{1}} \cos \theta_{x_{1}}+\varepsilon_{z x} \delta_{x x}+\varepsilon_{x x} \delta_{z y} \\
& -\varepsilon_{z x} \delta_{z y}-\varepsilon_{z x} \delta_{z x_{1}}+\varepsilon_{z y} \delta_{x z_{1}}+\varepsilon_{x x} \delta_{z x} \sin \theta_{x_{1}} \\
& -\varepsilon_{x y} \delta_{y_{1} z_{1}} \sin \theta_{x_{1}}+\varepsilon_{x x} \delta_{z_{1} z_{1}} \cos \theta_{x_{1}}-\varepsilon_{x y} \delta_{z_{1} z_{1}} \cos \theta_{x_{1}} \\
& -y \varepsilon_{x x}^{2}+z_{1} \varepsilon_{x x}^{2} \sin \theta_{x 1}+Z_{4} \varepsilon_{x x}^{2} \sin \theta_{x 1}-x \varepsilon_{x x} \varepsilon_{y x} \\
& +z_{1} \varepsilon_{y x} \varepsilon_{z x} \cos \theta_{x_{1}}+Z_{4} \varepsilon_{y x} \varepsilon_{z x} \cos \theta_{x_{1}}+z_{1} \varepsilon_{z x}^{2} \sin \theta_{x_{1}} \\
& +Z_{4} \varepsilon_{z x}^{2} \sin \theta_{x_{1}}-z_{1} \varepsilon_{x x} \varepsilon_{x y} \sin \theta_{x_{1}}-Z_{4} \varepsilon_{x x} \varepsilon_{x y} \sin \theta_{x_{1}} \\
& -y \varepsilon_{z x}^{2}-z_{1} \varepsilon_{z x} \varepsilon_{y y} \cos \theta_{x_{1}}-Z_{4} \varepsilon_{z x} \varepsilon_{y y} \cos \theta_{x_{1}} \\
& -z_{1} \varepsilon_{z x} \varepsilon_{z y} \sin \theta_{x_{1}}-Z_{4} \varepsilon_{z x} \varepsilon_{z y} \sin \theta_{x_{1}}-Z_{4} \varepsilon_{x x} \varepsilon_{x z_{1}} \sin \theta_{x_{1}} \\
& +Z_{4} \varepsilon_{x y} \varepsilon_{x z_{1}} \sin \theta_{x_{1}}-Z_{4} \varepsilon_{x z} \varepsilon_{y_{1} z_{1}}-Z_{4} \varepsilon_{z y} \varepsilon_{y_{1} z_{1}}+y \varepsilon_{z x} S_{x y} \\
& +z_{1} \varepsilon_{z x} S_{x z_{1}}-z_{1} \varepsilon_{z y} S_{x z_{1}}-z_{1} \varepsilon_{x x} S_{y_{1} z_{1}} \sin \theta_{x_{1}} \\
& \left.+z_{1} \varepsilon_{x y} S_{y_{1} z_{1}} \sin \theta_{x_{1}}\right) /\left(1+\varepsilon_{x x}^{2}+\varepsilon_{y x}^{2}+\varepsilon_{z x}^{2}\right) \\
& P_{z_{1}}=\left(y \varepsilon_{x x}-x \varepsilon_{y x}-\delta_{z x}+z_{1} \varepsilon_{x x} \sin \theta_{x_{1}}\right. \\
& -z_{1} \varepsilon_{x y} \sin \theta_{x_{1}}-Z_{4} \varepsilon_{x y} \sin \theta_{x_{1}}+\delta_{z y}-Z_{4} \varepsilon_{x z_{1}} \sin \theta_{x_{1}} \\
& +\delta_{y_{1} z_{1}} \sin \theta_{x_{1}}+\delta_{z_{1} z_{1}} \cos \theta_{x_{1}}-z_{1} S_{y_{1} z_{1}} \sin \theta_{x_{1}}-\varepsilon_{y x} \delta_{x x} \\
& +\varepsilon_{x x} \delta_{x y}-\varepsilon_{x x} \delta_{y y}+\varepsilon_{y x} \delta_{x z_{1}}-\varepsilon_{y y} \delta_{y_{1} z_{1}} \cos \theta_{x_{1}} \\
& +\varepsilon_{x y} \delta_{y_{1} z_{1}} \cos \theta_{x_{1}}+\varepsilon_{x x} \delta_{z_{1} z_{1}} \sin \theta_{x_{1}}-\varepsilon_{x y} \delta_{z_{1} z_{1}} \sin \theta_{x_{1}}
\end{aligned}
$$

$-z_{1} \varepsilon_{x x}^{2} \cos \theta_{x_{1}}-Z_{4} \varepsilon_{x x}^{2} \cos \theta_{x_{1}}$

$-z_{1} \varepsilon_{y x}^{2} \cos \theta_{x_{1}}-Z_{4} \varepsilon_{y x}^{2} \cos \theta_{x_{1}}-x \varepsilon_{x x} \varepsilon_{z x}+y \varepsilon_{z x}$

$-Z_{4} \varepsilon_{y x} \varepsilon_{z x} \sin \theta_{x_{1}}+z_{1} \varepsilon_{x x} \varepsilon_{x y} \cos \theta_{x_{1}}+Z_{4} \varepsilon_{x x} \varepsilon_{x y} \cos \theta_{x_{1}}$

$+z_{1} \varepsilon_{y x} \varepsilon y y \cos \theta_{x_{1}}+Z_{4} \varepsilon_{y x} \varepsilon_{y y} \cos \theta_{x_{1}}+z_{1} \varepsilon_{y x} \varepsilon_{z y} \sin \theta_{x_{1}}$

$+Z_{4} \varepsilon_{y x} \varepsilon_{z y} \sin \theta_{x_{1}}+Z_{4} \varepsilon_{x x} \varepsilon_{x z_{1}} \cos \theta_{x_{1}}-z_{1} \varepsilon_{y x} \varepsilon_{z x} \sin \theta_{x-1}$

$-Z_{4} \varepsilon_{x y} \varepsilon_{x z_{1}} \cos \theta_{x_{1}}+Z_{4} \varepsilon_{y x} \varepsilon_{y_{1} z_{1}}-Z_{4} \varepsilon_{y y} \varepsilon_{y_{1} z_{1}}-y \varepsilon_{y x} S_{x y}$

$-z_{1} \varepsilon_{y x} S_{x z_{1}}+z_{1} \varepsilon_{y y} S_{x z_{1}}+z_{1} \varepsilon_{x x} S_{y_{1} z_{1}} \cos \theta_{x_{1}}$

$\left.-z_{1} \varepsilon_{x y} S_{y_{1} z_{1}} \cos \theta_{x_{1}}\right) /\left(1+\varepsilon_{x x}^{2}+\varepsilon_{y x}^{2}+\varepsilon_{z x}^{2}\right)$

$P_{y_{2}}=\left(\delta_{y x}+z_{2} y \varepsilon_{x x} \cos \theta_{x_{2}}+Z_{6} \varepsilon_{x x} \cos \theta_{x_{2}}+x \varepsilon_{z x}-z_{2} \varepsilon_{x y} \cos \theta_{x_{2}}\right.$

$-Z_{6} \varepsilon_{x y} \cos \theta_{x_{2}}+\delta_{y y}-Z_{6} \varepsilon_{x z_{2}} \cos \theta_{x_{2}}+\delta_{y_{2} z_{2}} \cos \theta_{x_{2}}$

$-\delta_{z_{2} z_{2}} \sin \theta_{x_{2}}-z_{2} S_{y_{2} z_{2}} \cos \theta_{x_{2}}+\varepsilon_{z x} \delta_{x x}+\varepsilon_{x x} \delta_{z y}$

$-\varepsilon_{z x} \delta_{z y}-\varepsilon_{z x} \delta_{z x_{1}}+\varepsilon_{z y} \delta_{x z_{2}}+\varepsilon_{x x} \delta_{z x} \sin \theta_{x_{2}}$

$-\varepsilon_{x y} \delta_{y_{2} z_{2}} \sin \theta_{x_{2}}+\varepsilon_{x x} \delta_{z_{2} z_{2}} \cos \theta_{x_{2}}-\varepsilon_{x y} \delta_{z_{2} z_{2}} \cos \theta_{x_{2}}$

$-y \varepsilon_{x x}^{2}+z_{2} \varepsilon_{x x}^{2} \sin \theta_{x 1}+Z_{6} \varepsilon_{x x}^{2} \sin \theta_{x 1}-x \varepsilon_{x x} \varepsilon_{y x}$

$+z_{2} \varepsilon_{y x} \varepsilon_{z x} \cos \theta_{x_{2}}+Z_{6} \varepsilon_{y x} \varepsilon_{z x} \cos \theta_{x_{2}}+z_{2} \varepsilon_{z x}^{2} \sin \theta_{x_{2}}$

$+Z_{6} \varepsilon_{z x}^{2} \sin \theta_{x_{2}}-z_{2} \varepsilon_{x x} \varepsilon_{x y} \sin \theta_{x_{2}}-Z_{6} \varepsilon_{x x} \varepsilon_{x y} \sin \theta_{x_{2}}$

$-y \varepsilon_{z x}^{2}-z_{2} \varepsilon_{z x} \varepsilon_{y y} \cos \theta_{x_{2}}-Z_{6} \varepsilon_{z x} \varepsilon_{y y} \cos \theta_{x_{2}}$

$-z_{2} \varepsilon_{z x} \varepsilon_{z y} \sin \theta_{x_{2}}-Z_{6} \varepsilon_{z x} \varepsilon_{z y} \sin \theta_{x_{2}}-Z_{6} \varepsilon_{x x} \varepsilon_{x z_{2}} \sin \theta_{x_{2}}$

$+Z_{6} \varepsilon_{x y} \varepsilon_{x z_{2}} \sin \theta_{x_{2}}-Z_{6} \varepsilon_{x z} \varepsilon_{y_{2} z_{2}}-Z_{6} \varepsilon_{z y} \varepsilon_{y_{2} z_{2}}+y \varepsilon_{z x} S_{x y}$

$+z_{2} \varepsilon_{z x} S_{x z_{2}}-z_{2} \varepsilon_{z y} S_{x z_{2}}-z_{2} \varepsilon_{x x} S_{y_{2} z_{2}} \sin \theta_{x_{2}}$

$\left.+z_{2} \varepsilon_{x y} S_{y_{2} z_{2}} \sin \theta_{x_{2}}\right) /\left(1+\varepsilon_{x x}^{2}+\varepsilon_{y x}^{2}+\varepsilon_{z x}^{2}\right)$

$P_{z_{2}}=\left(y \varepsilon_{x x}-x \varepsilon_{y x}-\delta_{z x}+z_{2} \varepsilon_{x x} \sin \theta_{x_{2}}\right.$

$-z_{2} \varepsilon_{x y} \sin \theta_{x_{2}}-Z_{6} \varepsilon_{x y} \sin \theta_{x_{2}}+\delta_{z y}-Z_{6} \varepsilon_{x z_{2}} \sin \theta_{x_{2}}$

$+\delta_{y_{1} z_{2}} \sin \theta_{x_{2}}+\delta_{z_{2} z_{2}} \cos \theta_{x_{2}}-z_{2} S_{y_{1} z_{2}} \sin \theta_{x_{2}}-\varepsilon_{y x} \delta_{x x}$

$+\varepsilon_{x x} \delta_{x y}-\varepsilon_{x x} \delta_{y y}+\varepsilon_{y x} \delta_{x z_{2}}-\varepsilon_{y y} \delta_{y_{1} z_{2}} \cos \theta_{x_{2}}$ 


$$
\begin{aligned}
& +\varepsilon_{x y} \delta_{y_{1} z_{2}} \cos \theta_{x_{2}}+\varepsilon_{x x} \delta_{z_{2} z_{2}} \sin \theta_{x_{2}}-\varepsilon_{x y} \delta_{z_{2} z_{2}} \sin \theta_{x_{2}} \\
& -z_{2} \varepsilon_{x x}^{2} \cos \theta_{x_{2}}-Z_{6} \varepsilon_{x x}^{2} \cos \theta_{x_{2}} \\
& -z_{2} \varepsilon_{y x}^{2} \cos \theta_{x_{2}}-Z_{6} \varepsilon_{y x}^{2} \cos \theta_{x_{2}}-x \varepsilon_{x x} \varepsilon_{z x}+y \varepsilon_{z x} \\
& -Z_{6} \varepsilon_{y x} \varepsilon_{z x} \sin \theta_{x_{2}}+z_{2} \varepsilon_{x x} \varepsilon_{x y} \cos \theta_{x_{2}}+Z_{6} \varepsilon_{x x} \varepsilon_{x y} \cos \theta_{x_{2}} \\
& +z_{2} \varepsilon_{y x} \varepsilon y y \cos \theta_{x_{2}}+Z_{6} \varepsilon_{y x} \varepsilon_{y y} \cos \theta_{x_{2}}+z_{2} \varepsilon_{y x} \varepsilon_{z y} \sin \theta_{x_{2}} \\
& +Z_{6} \varepsilon_{y x} \varepsilon_{z y} \sin \theta_{x_{2}}+Z_{6} \varepsilon_{x x} \varepsilon_{x z_{2}} \cos \theta_{x_{2}}-z_{2} \varepsilon_{y x} \varepsilon_{z x} \sin \theta_{x-1} \\
& -Z_{6} \varepsilon_{x y} \varepsilon_{x z_{2}} \cos \theta_{x_{2}}+Z_{6} \varepsilon_{y x} \varepsilon_{y_{1} z_{2}}-Z_{6} \varepsilon_{y y} \varepsilon_{y_{1} z_{2}}-y \varepsilon_{y x} S_{x y} \\
& -z_{2} \varepsilon_{y x} S_{x z_{2}}+z_{2} \varepsilon_{y y} S_{x z_{2}}+z_{2} \varepsilon_{x x} S_{y_{1} z_{2}} \cos \theta_{x_{2}} \\
& \left.-z_{2} \varepsilon_{x y} S_{y_{1} z_{2}} \cos \theta_{x_{2}}\right) /\left(1+\varepsilon_{x x}^{2}+\varepsilon_{y x}^{2}+\varepsilon_{z x}^{2}\right)
\end{aligned}
$$

Acknowledgement Presented work has been supported in part by the Ministry of Commerce, Industry and Energy, Korea and the University of Michigan NSF Engineering Research Center for Reconfigurable Manufacturing Systems (NSF grant \# EEC-9529125).

\section{References}

1. Ozdoganlar OB (1999) Stability of single- and parallel-process machining including geometry of corner-radiused tooling. Dissertation, The University of Michigan, Mechanical Engineering, Ann Arbor

2. Roberts R (2000) High-speed holemaking. Manuf Eng 125(1):108-112

3. Ozdoganlar OB, Endres WJ (1999) Parallel-process (simultaneous) machine and its stability. MED Manuf Sci Eng 10:361-368

4. Koren Y, Kota S (1999) Reconfigurable machine tools. USA Patent \#5943750
5. Landers RG, Min B-K, Koren Y (2001) Reconfigurable machine tools. Ann CIRP 50(1):269-274

6. Spicer P, Koren Y, Shpitalni M, Yip-Hoi D (2002) Design principles for machining system configurations. Ann CIRP 51(1):275-280

7. Lorincz J (2001) Flexible production wins auto game. Tool Prod 67(1):48-52

8. Owen JV (1995) Multispindles multiply. Manuf Eng 114(5):47

9. Vorob'eva TS (1971) Vibration in multi-spindle vertical semi-automatic lathes. Mach Tool 42:29-33

10. Lazoglu I, Volger M, Kapoor SG, DeVor RE (1998) Dynamics of the simultaneous turning process. Trans NAMRI SME 26:135-140

11. Bartalucci B, Bedini R, Lisini GG (1971) Optimization of manufacturing on multi-spindle machines. Ann CIRP 19(1):602-605

12. Jain C, Hancook WM (1976) Failure characterization of multi-spindle multi-station machines. Int J Prod Res 14:711-717

13. Okafor AC, Ertekin YM (2000) Derivation of machine tool error models and error compensation procedure for three axes vertical machining center using rigid body kinematics. Int J Mach Tools Manuf 40(8):1199-1213

14. Ahn KG, Cho DW (1999) Proposition for a volumetric error model considering backlash in machine tools. Int J Adv Manuf Technol 15: 554-561

15. Chen JS, Yuan JX, Ni J, Wu SM (1993) Real-time compensation for time-variant volumetric errors on a machining center. Trans ASME, J Eng Ind 114(4):472-479

16. Eman KF, Wu BT (1987) A generalized geometric error model for multi-axis machines. Ann CIRP 36(1):253-256

17. Ferreira PM, Liu CR (1986) An analytical quadratic model for the geometric error of a machine tool. J Manuf Syst 5(1):51-63

18. Wang $H$ (2004) Identification of strut and assembly errors of a 3-PRS serial-parallel machine tool. Int J Mach Tools Manuf 44(11):1171-1178

19. Weck M (1980) Handbook of machine tools vol. 4 Metrological analysis and performance test, (English translation). Wiley, New York 